\title{
Fagfellevurderere 2012
}

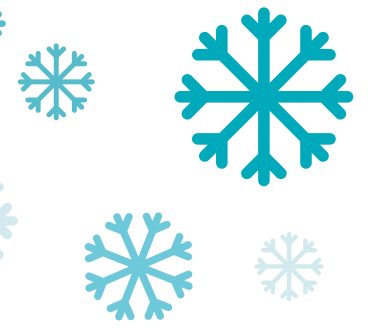

Alstadhaug, Karl B. Anfinsen, Ole-Gunnar Angelsen, Anders Aurlien, Dag Aurlien, Harald

Austad, Joar

Bachmann, Ingeborg

Backe, Bjørn

Bahus, Marianne

Bakke, Søren

Balteskard, Lise

Bartnes, Kristian

Becker, Frank

Bekkelund, Svein

Bell, Rae

Bendiksen, Cecilie

Benestad, Haakon

Berentsen, Sigbjørn

Berg, Tore Julsrud

Bergan, Stein

Berge, Eivind

Berge, Rolf Kristian

Berge, Viktor

Bergem, Astrid Liv Mina

Berild, Dag

Berner, Aasmund

Berntsen, Erik Magnus

Bertelsen, Bjørn

Birkeland, Kåre

Bjordal, Jan

Bjørge, Line

Bjørgul, Kristian

Bjørnelv, Sigrid

Bjørner, Trine

Blom, Hans

Bosse, Gerhard

Braarud, Anne Cathrine

Bramness, Jørgen

Brandsaeter, Bjorn

Brandstorp, Helen

Brantsæter, Arne

Brattebø, Guttorm

Braut, Geir

Brautaset, Nils

Breivik, Harald
Bretthauer, Michael

Brokstad, Karl

Brunvand, Leif

Brustugun, Odd Terje

Bruun Wyller, Vegard

Bruun, Johan

Bruusgaard, Dag

Brækhus, Anne

Bugge, Jan

Bærheim, Anders

Calisch, Tor Einar

Celius, Elisabeth

Claudi, Tor

Claussen, Bjørgulf

Dahl, Alv

Dale, Ola

Dalgaard, Jakob

de Weerd, Louis

Diaz, Esperanza

Due, Jan

Døllner, Henrik

Ebbing, Cathrine

Edvardsen, Thor

Eggen, Anne Elise

Ekeberg, Øivind

Ekerhovd, Erling

Ellingsen, Christian

Elverland, Hans

Engedal, Knut

Engelsen, Bernt

Eri, Lars

Eriksen, Hanne

Eskild, Anne

Evensen, Stein

Falhof, Jannik

Finsen, Vilhjalmur

Finset, Arnstein

Flaatten, Hans

Flaten, Magne

Flægstad, Trond

Fosse, Erik

Fosse, Roar

Fretheim, Atle

Frich, Jan

Frøen, Frederik

Furnes, Ove

Fønnebø, Vinjar

Førde, Reidun

Gadeholt, Gaut

Garcia, Beate

Gisvold, Sven Erik

Giæver, Anton

Gjersing, Linn
Gjerstad, Leif

Gjesdal, Knut

Gjessing, Håkon

Gordeladze, Jan

Gradmann, Christoph

Graff-Iversen, Sidsel

Gramstad, Lars

Gran, Jan

Gran, Sarah

Grundvold, Irene

Grøholt, Berit

Grønn, Morten

Gudim, Hilde Beate

Gulbrandsen, Pål

Gulseth, Hanne

Haagaas, Ivar

Haarr, Dagfinn

Haavaldsen, Camilla

Haave, Per

Hagen, Bjørn

Hagve, Tor-Arne

Halvorsen, Bente

Hammar, Åsa

Hannestad, Yngvild

Hansen, John-Bjarne

Hansen, Thor Willy Ruud

Hartmann, Anders

Hasle, Gunnar

Hatlebakk, Jan

Haug, Jon

Heier, Hans

Helgeland, Lars

Helland, Åslaug

Helseth, Eirik

Helsing, Per

Hem, Erlend

Herfjord, Jo Kåre

Hernes, Eivor

Hilt, Bjørn

Hjelmesæth, Jøran

Hjørleifsson, Stefan

Hoff, Jana

Hoff, Jon Erik

Hoff-Olsen, Per

Hofmann, Bjørn

Hofstad, Bjørn

Holmøy, Trygve

Holst, Dorthe

Houge, Gunnar

Hovland, Randi

Humerfelt, Sjur

Hunskår, Steinar

Husby, Torstein

Husebekk, Anne

Husebye, Eystein

Hyldmo, Per Kristian

Høymork, Siv Cathrine
Ilner, Stein Opjordsmoen

Indahl, Aage

Indredavik, Bent

Irtun, Øivind

Iversen, Ole-Erik

Jacobsen, Dag

Jacobsen, Eva

Jacobsson, Bo

Jakobsen, Jarl

Jakola, Asgeir

Jensen, Anders Ditlev

Johansen, Lars

Johansen, Rune

Johansen, Truls

Juel, Niels Gunnar

Jørgensen, Anders Palmstrøm

Kalager, Mette

Kerty, Emilia

Kessler, Jörg

Kielland, Knut

Kinge, Bettina

Kiserud, Torvid

Klepp, Knut-Inge

Knut Eirik, Eliassen

Koch, Anne Mette

Kongsgaard, Ulf

Kostov, Hrisimir

Kristensen, Øistein

Kristiansen, Ivar Sønbø

Krohn, Jørgen

Kvande, Lise

Kvarstein, Gunnvald

Kvistad, Kjell

Kværner, Kari

Kvåle, Reidar

Kvåle, Rune

Kyte, Jon Amund

Kørner, Hartwig

Lang, Astri Maria

Langslet, Gisle

Larsen, Alf

Larsen, Bent

Lassen, Kristoffer

Leira, Håkon Lasse

Lereim, Inggard

Lie, Birgit

Linaker, Olav

Lindbæk, Morten

Lindemann, Rolf

Ljøstad, Unn

Loge, Jon Håvard

Lossius, Hans Morten

Lossius, morten

Lund, Caroline

Lund, Christian 
Lund, Eiliv

Lundgren, Steinar

Lundin, Knut

Løkeland, Mette

Lømo, Terje

Madsen, Steinar

Magelssen, Morten

Magnussen, Jon

Malterud, Kirsti

Marienhagen, Kirsten

Markestad, Trond J.

Meberg, Alf

Mella, Olav

Melle, Ingrid

Mellgren, Svein

Middelthon, Anne-Lise

Midelfart, Anna

Moen, Mette

Morken, Gunnar

Moseng, Ole Georg

Myhr, Kjell-Morten

Mæhle, Bjørn Ove

Nakken, Karl Otto

Nakstad, Per

Nessa, John

Nielsen, Erik Waage

Nilsen, Kristian

Nordbø, Svein Arne

Nordeng, Hedvig

Nordrehaug, Jan Erik

Norheim, Ingrid

Norheim, Ole Frithjof

Nortvedt, Per

Norum, Kaare

Nygaard, Øystein

Næss, Halvor

Næss, Pål Aksel

Oldervoll, Line Merethe

Olweus, Johanna

Omvik, Per

Opdal, Mimi

Ose, Leiv

Paulssen, Eyvind

Paus, Benedicte

Pettersen, Renate

Pihlstrøm, Lasse

Raeder, Sten

Raknes, Guttorm

Ranhoff, Anette

Reiso, Harald

Rekand, Tiina

Retterstøl, Kjetil

Riedel, Bettina
Risøe, Cecilie

Robsahm, Trude

Rogde, Sidsel

Rosenqvist, Randi

Rosenvinge, Jan $\mathrm{H}$.

Rosness, Tor

Rosseland, Leiv Arne

Ræder, Johan

Rønning, Ole Morten

Rørtveit, Guri

Sager, Georg

Sagsveen, Mette

Salvesen, Rolf

Salvesen, Kjell

Sand, Trond

Sandanger, Inger

Sandberg, Mårten

Sandset, Per Morten

Sauer, Torill

Schiøtz, Aina

Selbæk, Geir

Sevre, Knut

Simonsen, Gunnar Skov

Simonsen, Anne

Skatvedt, Olav

Skjeldestad, Finn Egil

Skrede, Steinar

Skålevåg, Svein Atle

Skålhegg, Bjørn

Slørdal, Lars

Småbrekke, Lars

Solberg, Berge

Sorteberg, Angelika

Specht, Karsten

Spigset, Olav

Staff, Annetine

Steen, Petter Andreas

Steigen, Sonja

Steinsbekk, Aslak

Steinsvåg, Sverre

Stensvold, Einar

Stovner, Lars Jacob

Strøm, Rune Tore

Strømme, Petter

Stugaard, Marie

Sundby, Johanne

Sundstrøm, Stein H.

Svensson, Olle

Søreide, Kjetil

Taubøll, Erik

Tesli, Martin

Thomassen, Lars

Thommessen, Bente

Tjølsen, Arne

Torgersen, Terje

Tretli, Steinar
Tveit, Arnljot

Tysnes, Ole-Bjørn

Uhlig, Till

Uhlin-Hansen, Lars

Ulvestad, Elling

Ulvik, Rune

Vaaler, Arne

Vandvik, Inger Helene

Varhaug, Jan Erik

Vatten, Lars

Vedeler, Christian

Viste, Asgaut

Vollset, Stein Emil

Waal, Helge

Warncke, Torhild

Wesenberg, Finn

Wester, Knut Gustav

Westin, Andreas

Wien, Tale

Willoch, Frode

Winther, Rolf B.

Wyller, Torgeir

Zahl, Per-Henrik

Zeiner, Pål

Zwart, John-Anker

Øian, Pål

Øiesvold, Terje

Ørstavik, Kristin

Aamdal, Steinar

Aamodt, Geir

Aanonsen, Nils

Aasheim, Erlend

Aasland, Olaf

Aasly, Jan

Aavitsland, Preben

Åsberg, Anders

Åsjö, Birgitta 